\title{
Chromium(II)-catalyzed enantioselective arylation of ketones
}

\author{
Gang Wang ${ }^{1,2}$, Shutao Sun ${ }^{2}$, Ying Mao ${ }^{2}$, Zhiyu Xie $^{2}$ and Lei Liu ${ }^{* 1,2,3}$
}

\author{
Full Research Paper \\ Address: \\ ${ }^{1}$ Shenzhen Research Institute of Shandong University, Shenzhen \\ 518057, China, ${ }^{2}$ School of Pharmaceutical Sciences, Shandong \\ University, Jinan 250012, China and ${ }^{3}$ School of Chemistry and \\ Chemical Engineering, Shandong University, Jinan 250100, China \\ Email: \\ Lei Liu* - leiliu@sdu.edu.cn \\ * Corresponding author \\ Keywords: \\ arylation; asymmetric catalysis; chromium; ketone; tertiary alcohol
}

Open Access

Beilstein J. Org. Chem. 2016, 12, 2771-2775.

doi:10.3762/bjoc. 12.275

Received: 17 October 2016

Accepted: 06 December 2016

Published: 19 December 2016

This article is part of the Thematic Series "Strategies in asymmetric catalysis".

Guest Editor: T. P. Yoon

() 2016 Wang et al.; licensee Beilstein-Institut.

License and terms: see end of document.

\begin{abstract}
The chromium-catalyzed enantioselective addition of carbo halides to carbonyl compounds is an important transformation in organic synthesis. However, the corresponding catalytic enantioselective arylation of ketones has not been reported to date. Herein, we report the first $\mathrm{Cr}$-catalyzed enantioselective addition of aryl halides to both arylaliphatic and aliphatic ketones with high enantioselectivity in an intramolecular version, providing facile access to enantiopure tetrahydronaphthalen-1-ols and 2,3-dihydro-1 $H$ inden-1-ols containing a tertiary alcohol.
\end{abstract}

\section{Introduction}

Catalytic enantioselective carbon-carbon bond formation reactions have achieved enormous development during the last few decades as a consequence of the growing demand for enantiopure compounds in modern industry, especially the pharmaceutical industry. The chromium ( $\mathrm{Cr}$ )-catalyzed enantioselective addition of carbo halides to carbonyl compounds is one of the most reliable methods in organic chemistry for chemoselective and structurally diverse synthesis [1-9]. To date, the Cr-catalyzed enantioselective carbonyl addition reactions mainly focused on allylation, propargylation, alkenylation and alkylation of aldehydes [10,11]. Since the first example of enantioselective allylation of aldehydes catalyzed by a Cr(II)-salen complex in 1999 by Cozzi and co-workers [12], several elegant cat- alytic enantioselective allylation and propargylation reactions have been developed by the groups of Nakada [13,14], Berkessel [15], Kishi [16], Sigman [17], Yamamoto [18], Guiry [19], Chen [20], Gade [21], White [22], and Zhang [23-25], respectively. The alkenylation and alkylation reactions were mainly explored by the Kishi group [26-30], and they established a toolbox approach to search for the specific ligand with a given substrate in the Cr-catalyzed process [28]. They successfully applied the method to the natural product total synthesis like halichondrin B and norhalichondrin B, and in the subsequent pharmaceutical study, finally leading to the discovery of the anticancer drug Eribulin [31-35]. However, to our knowledge, the Cr-catalyzed enantioselective arylation of carbonyl 
compounds has rarely been explored. On the other hand, most of the reactions focused on aldehyde components, while asymmetric addition to ketones remains a big challenge probably due to the decreased reactivity and selectivity [36,37]. A breakthrough was made by the Sigman group who reported the catalytic enantioselective addition of allylic bromides and propargyl halides to arylaliphatic ketones using oxazoline ligands with high enantioselectivity (up to 95\% ee) [38-41]. After that, the Chen group also disclosed enantioselective allylation of ketones using spirocyclic chiral borate and chiral bipyridyl alcohol ligands with the ee value ranging from $27 \%$ to $97 \%[42,43]$. However, as far as we know, a Cr-catalyzed enantioselective arylation of ketones has never been reported to date [44]. Tetrahydronaphthalen-1-ol bears a chiral tertiary alcohol center and is a common structural motif in numerous biologically active natural products and clinical drugs [45]. The method to prepare these compounds through intramolecular arylation of ketones would be highly desired. Herein, we report the first $\mathrm{Cr}$-catalyzed enantioselective arylation of ketones in an intramolecular version.

\section{Results and Discussion}

Initially, the Cr-catalyzed asymmetric intramolecular arylation of arylaliphatic ketone 5-(2-iodophenyl)pentan-2-one (1a) was selected as the model reaction for optimization employing Kishi's oxazoline/sulfonamides as the chiral ligands. A series of oxazoline/sulfonamide ligands (L1-L8) were tested and the results were summarized in Table 1 . Four subgroups of $\mathrm{R}^{1}$ were studied (entries 1-4, Table 1) and isopropyl substituted oxazoline proved to be the best ligand with a $42 \%$ ee. Afterwards, $\mathrm{R}^{2}$ (Table 1, entries 2, 5 and 6) and $\mathrm{R}^{3}$ (Table 1, entries 6-8) substituents were also examined, and $\mathbf{L 8}$ bearing a methyl

Table 1: Screening conditions of the catalytic enantioselective Cr-mediated arylation of ketone. ${ }^{a}$

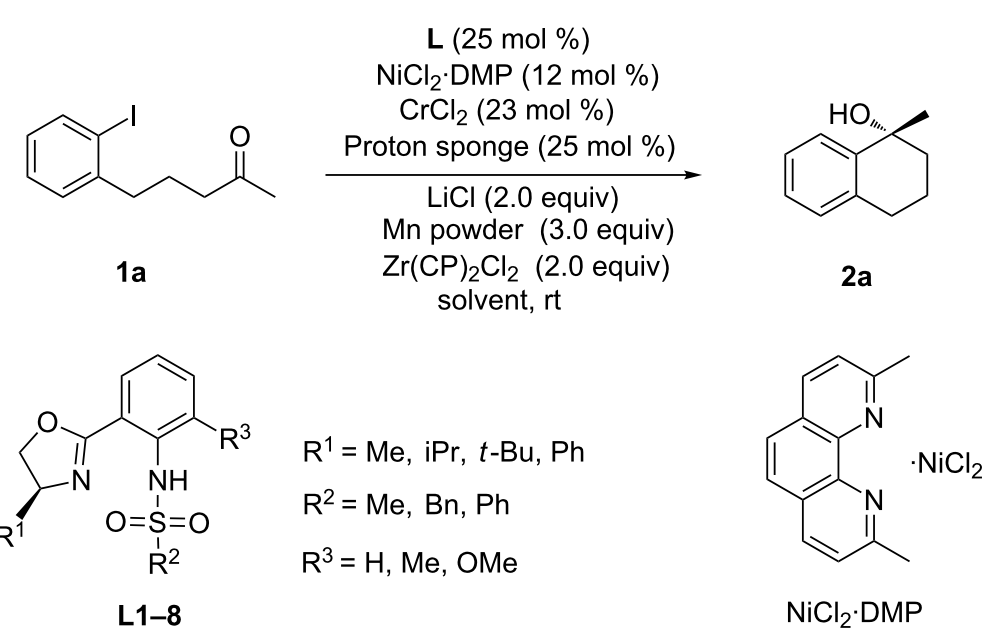

\begin{tabular}{|c|c|c|c|c|c|c|c|}
\hline entry & $\mathbf{L}$ & $\mathrm{R}^{1}$ & $\mathrm{R}^{2}$ & $\mathrm{R}^{3}$ & Solvent & Yield (\%)a & $e e^{b}$ \\
\hline 1 & L1 & $\mathrm{Me}$ & $\mathrm{Ph}$ & $\mathrm{H}$ & $\mathrm{MeCN}$ & 43 & 28 \\
\hline 2 & L2 & $\mathrm{iPr}$ & $\mathrm{Ph}$ & $\mathrm{H}$ & $\mathrm{MeCN}$ & 50 & 42 \\
\hline 3 & L3 & $t-\mathrm{Bu}$ & $\mathrm{Ph}$ & $\mathrm{H}$ & $\mathrm{MeCN}$ & 47 & 25 \\
\hline 4 & L4 & $\mathrm{Ph}$ & $\mathrm{Ph}$ & $\mathrm{H}$ & $\mathrm{MeCN}$ & 51 & 21 \\
\hline 5 & L5 & $\mathrm{iPr}$ & $\mathrm{Bn}$ & $\mathrm{H}$ & $\mathrm{MeCN}$ & 12 & 37 \\
\hline 6 & L6 & $\mathrm{iPr}$ & $\mathrm{Me}$ & $\mathrm{H}$ & $\mathrm{MeCN}$ & 49 & 56 \\
\hline 7 & L7 & $\mathrm{iPr}$ & $\mathrm{Me}$ & OMe & $\mathrm{MeCN}$ & 75 & 75 \\
\hline 8 & L8 & $\mathrm{iPr}$ & $\mathrm{Me}$ & $\mathrm{Me}$ & $\mathrm{MeCN}$ & 85 & 82 \\
\hline 9 & L8 & $\mathrm{iPr}$ & $\mathrm{Me}$ & $\mathrm{Me}$ & THF & 93 & 81 \\
\hline 10 & L8 & $\mathrm{iPr}$ & $\mathrm{Me}$ & $\mathrm{Me}$ & DME & 90 & 86 \\
\hline $11^{\mathrm{c}}$ & L8 & $\mathrm{iPr}$ & $\mathrm{Me}$ & $\mathrm{Me}$ & DME & 86 & 92 \\
\hline $12^{d}$ & L8 & $\mathrm{iPr}$ & $\mathrm{Me}$ & $\mathrm{Me}$ & DME & 81 & 97 \\
\hline $13^{e}$ & L8 & $\mathrm{iPr}$ & $\mathrm{Me}$ & $\mathrm{Me}$ & DME & 51 & 83 \\
\hline $14^{\mathrm{d}, \mathrm{f}}$ & L8 & $\mathrm{iPr}$ & $\mathrm{Me}$ & $\mathrm{Me}$ & DME & 35 & 70 \\
\hline
\end{tabular}

aYield of isolated product. betermined by HPLC analysis on a chiral column. ${ }^{\mathrm{c}}$ Reaction at $0{ }^{\circ} \mathrm{C}$ for $24 \mathrm{~h}$. ${ }^{\mathrm{d}}$ Reaction at $-20{ }^{\circ} \mathrm{C}$ for $24 \mathrm{~h}$. e Reaction at $-40{ }^{\circ} \mathrm{C}$ for $24 \mathrm{~h}$. ${ }^{\mathrm{A}} \mathrm{Aryl}$ bromide used instead of aryl iodide. 
group in both $\mathrm{R}^{2}$ and $\mathrm{R}^{3}$ gave the best enantiocontrol. The solvent effect was then investigated, and 1,2-dimethoxyethane (DME) was identified to be the best choice (Table 1, entries $8-10)$. Lowering the reaction temperature was found to be beneficial for improving the enantioselectivity, and when the reaction was performed at $-20{ }^{\circ} \mathrm{C}$, expected $\mathbf{2 a}$ was isolated in $81 \%$ yield with $97 \%$ ee (Table 1, entries 10-13). Aryl bromide proved to be an inferior coupling component, providing $2 \mathbf{a}$ in $35 \%$ yield and $70 \%$ ee (Table 1 , entry 14 ).

With the optimized conditions in hand, the scope of the ketone component was first explored (Scheme 1). Aliphatic ketones with (longer) alkyl chain such as ethyl (1b) and $n$-hexyl ketones (1c), were also tolerated albeit with slightly decreased

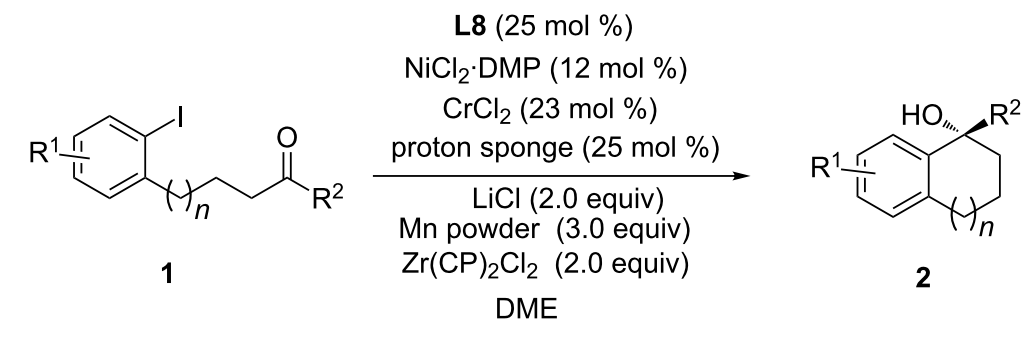<smiles>CC1(O)CCCc2ccccc21</smiles>

2a

$81 \%, 97 \%$ ee<smiles>OC1(c2ccccc2)CCCc2ccccc21</smiles>

2d

$65 \%, 88 \%$ ee<smiles>OC1(c2cccc(F)c2)CCCc2ccccc21</smiles>

$2 \mathrm{~g}$

$68 \%, 90 \%$ ee<smiles>Cc1cccc2c1CCCC2(C)O</smiles>

2j

$71 \%, 90 \%$ ee<smiles>C[C@]1(O)CCc2ccccc21</smiles>

$2 \mathrm{~m}$

$70 \%, 82 \%$ ee<smiles>CCC1(O)CCCc2ccccc21</smiles>

2b

$75 \%, 91 \%$ ee<smiles>COc1ccc([C@]2(O)CCCc3ccccc32)cc1</smiles>

$2 \mathrm{e}$

$52 \%, 78 \%$ ee<smiles>OC1(c2cc(C(F)(F)F)cc(C(F)(F)F)c2)CCCc2ccccc21</smiles>

2h

$62 \%, 86 \%$ ee<smiles>COc1ccc2c(c1)CCCC2(C)O</smiles>

2k

$74 \%, 88 \%$ ee<smiles>CCCCCC[C@]1(O)CCCc2ccccc21</smiles>

2c

$53 \%, 87 \%$ ee<smiles>OC1(c2ccc(Cl)cc2)CCCc2ccccc21</smiles>

$2 \mathbf{f}$

$64 \%, 90 \%$ ee<smiles>O[C@@]1(c2ccco2)CCCc2ccccc21</smiles>

$2 \mathbf{i}$

$63 \%, 78 \%$ ee<smiles>COc1cc2c(cc1OC)[C@](C)(O)CCC2</smiles>

2I

$76 \%, 88 \%$ ee 
yield and selectivity. The asymmetric arylation of various arylaliphatic ketones also went smoothly (1d-h). Phenyl ketone 1d and ketones with electron-withdrawing groups in different substituent patterns gave the expected products with good enantiocontrol, while the enantioselectivity for ketone $\mathbf{1 e}$ bearing an electron-donating group decreased. The mild process exhibited excellent functional group tolerance, with chloride (2f), fluoride (2g), and $\mathrm{CF}_{3}$ moieties (2h) well tolerated for further manipulation $[46,47]$. Heteroaryl ketones such as furansubstituted ketone (1i) were also suitable substrate, giving product $2 \mathbf{i}$ in $78 \%$ ee. The scope of the aryl halide component was next explored $(\mathbf{1} \mathbf{j}-\mathbf{l})$. Aryl halides bearing different substituent patterns were tolerated giving the tetrahydronaphthalen-1-ols with good ee values. When 4-(2-iodophenyl)butan-2-one (1m) was used, enantiopure indan-1-ol was obtained in $70 \%$ yield and $82 \%$ ee.

\section{Conclusion}

In summary, we have developed the first $\mathrm{Cr}$-catalyzed enantioselective arylation of ketones in an intramolecular version using oxazoline/sulfonamide $\mathbf{L 8}$ as the catalyst. Both aliphatic and arylaliphatic ketones proceeded smoothly, providing corresponding tetrahydronaphthalen-1-ols bearing a tertiary alcohol center with good enantioselectivities (up to $97 \%$ ee).

\section{Experimental}

General procedure for the chromium(II) catalyzed enantioselective arylation of ketones: The solution of $\mathbf{L 8}(0.25$ equiv, $0.025 \mathrm{mmol})$, proton sponge $(0.25$ equiv, $0.025 \mathrm{mmol})$ and $\mathrm{CrCl}_{2}(0.23$ equiv, $0.023 \mathrm{mmol})$ in DME $(1.0 \mathrm{~mL})$ was stirred at room temperature in a glove-box for $1 \mathrm{~h}$. Then the substrate 1 (1.0 equiv, $0.1 \mathrm{mmol}$ ), $\mathrm{LiCl}$ (2.0 equiv, $0.2 \mathrm{mmol}$ ), $\mathrm{Mn}$ powder (3.0 equiv, $0.3 \mathrm{mmol}), \mathrm{NiCl}_{2} \cdot \mathrm{DMP}(0.12$ equiv, $0.012 \mathrm{mmol})$ and $\mathrm{Zr}(\mathrm{CP})_{2} \mathrm{Cl}_{2}$ (2.0 equiv, $0.2 \mathrm{mmol}$ ) were added successively and the mixture was stirred at indicated temperature for $24 \mathrm{~h}$. After that, the mixture was filtered through a short pad of celite and purified by flash chromatography using silica gel or alumina (200-300 mesh) to give the product 2 .

\section{Supporting Information}

\section{Supporting Information File 1}

Experimental procedures, analytical data for products, copies of NMR spectra and HPLC chromatograms. [http://www.beilstein-journals.org/bjoc/content/ supplementary/1860-5397-12-275-S1.pdf]

\section{Acknowledgements}

We thank the National Science Foundation of China (21472112), Fok Ying Tung Education Foundation (151035), the Program for New Century Excellent Talents in University (NCET-13-0346), Shenzhen Strategic Emerging Industry Development Special Funds (JCYJ20150430160921949), the Shandong Science Fund for Distinguished Young Scholars (JQ201404), and the Fundamental Research Funds of Shandong University (2015JC035) for financial support.

\section{References}

1. Okude, Y.; Hirano, S.; Hiyama, T.; Nozaki, H. J. Am. Chem. Soc. 1977, 99, 3179-3181. doi:10.1021/ja00451a061

2. Okude, Y.; Hiyama, T.; Nozaki, H. Tetrahedron Lett. 1977, 18, 3829-3830. doi:10.1016/S0040-4039(01)83365-7

3. Fürstner, A.; Shi, N. J. Am. Chem. Soc. 1996, 118, 2533-2534. doi:10.1021/ja9537538

4. Fürstner, A.; Shi, N. J. Am. Chem. Soc. 1996, 118, 12349-12357. doi:10.1021/ja9625236

5. Avalos, M.; Babiano, R.; Cintas, P.; Jiménez, J. L.; Palacios, J. C. Chem. Soc. Rev. 1999, 28, 169-177. doi:10.1039/a806117c

6. Takai, K.; Nozaki, H. Proc. Jpn. Acad., Ser. B 2000, 76, 123-131. doi:10.2183/pjab.76.123

7. Fürstner, A. Chem. Rev. 1999, 99, 991-1046. doi:10.1021/cr9703360

8. Hargaden, G. C.; Guiry, P. J. Adv. Synth. Catal. 2007, 349, 2407-2424. doi:10.1002/adsc.200700324

9. Tian, Q.; Zhang, G. Synthesis 2016, 48, 4038-4049. doi:10.1055/s-0036-1589457

10. Choi, H.-w.; Nakajima, K.; Demeke, D.; Kang, F.-A.; Jun, H.-S.; Wan, Z.-K.; Kishi, Y. Org. Lett. 2002, 4, 4435-4438. doi:10.1021/ol026981x

11. Namba, K.; Kishi, Y. Org. Lett. 2004, 6, 5031-5033. doi:10.1021/ol047661b

12. Bandini, M.; Cozzi, P. G.; Melchiorre, P.; Umani-Ronchi, A. Angew. Chem. 1999, 111, 3558-3561. doi:10.1002/(SICI)1521-3757(19991115)111:22<3558::AID-ANGE3558 $>3.0 . \mathrm{CO} ; 2-6$

Angew. Chem. Int. Ed. 1999, 38, 3357-3359. doi:10.1002/(SICI)1521-3773(19991115)38:22<3357::AID-ANIE3357>3 .0.CO;2-W

13. Inoue, M.; Suzuki, T.; Nakada, M. J. Am. Chem. Soc. 2003, 125, 1140-1141. doi:10.1021/ja021243p

14. Inoue, M.; Nakada, M. Org. Lett. 2004, 6, 2977-2980. doi:10.1021/ol048826j

15. Berkessel, A.; Menche, D.; Sklorz, C. A.; Schröder, M.; Paterson, I. Angew. Chem. 2003, 115, 1062-1065. doi:10.1002/ange.200390240 Angew. Chem., Int. Ed. 2003, 42, 1032-1035. doi:10.1002/anie.200390265

16. Kurosu, M.; Lin, M.-H.; Kishi, Y. J. Am. Chem. Soc. 2004, 126, 12248-12249. doi:10.1021/ja045557j

17. Lee, J.-Y.; Miller, J. J.; Hamilton, S. S.; Sigman, M. S. Org. Lett. 2005, 7, 1837-1839. doi:10.1021/ol050528e

18. Xia, G.; Yamamoto, H. J. Am. Chem. Soc. 2006, 128, 2554-2555. doi:10.1021/ja058454p

19. McManus, H. A.; Cozzi, P. G.; Guiry, P. J. Adv. Synth. Catal. 2006, 348, 551-558. doi:10.1002/adsc.200505332

20. Huang, X.-R.; Pan, X.-H.; Lee, G.-H.; Chen, C. Adv. Synth. Catal. 2011, 353, 1949-1954. doi:10.1002/adsc.201100023

21. Deng, Q.-H.; Wadepohl, H.; Gade, L. H. Chem. - Eur. J. 2011, 17, 14922-14928. doi:10.1002/chem.201102375 
22. White, J. D.; Shaw, S. Org. Lett. 2011, 13, 2488-2491. doi:10.1021/ol2007378

23. Chen, W.; Yang, Q.; Zhou, T.; Tian, Q.; Zhang, G. Org. Lett. 2015, 17, 5236-5239. doi:10.1021/acs.orglett.5b02597

24. Xiong, Y.; Zhang, G. Org. Lett. 2016, 18, 5094-5097.

doi:10.1021/acs.orglett.6b02540

25. Tian, Q.; Bai, J.; Chen, B.; Zhang, G. Org. Lett. 2016, 18, 1828-1831. doi:10.1021/acs.orglett.6b00559

26. Namba, K.; Cui, S.; Wang, J.; Kishi, Y. Org. Lett. 2005, 7, 5417-5419. doi:10.1021/ol052084s

27. Namba, K.; Cui, S.; Wang, J.; Kishi, Y. Org. Lett. 2005, 7, 5421-5424. doi:10.1021/ol052085k

28. Guo, H.; Dong, C.-G.; Kim, D.-S.; Urabe, D.; Wang, J.; Kim, J. T.; Liu, X.; Sasaki, T.; Kishi, Y. J. Am. Chem. Soc. 2009, 131, 15387-15393. doi:10.1021/ja905843e

29. Liu, X.; Li, X.; Chen, Y.; Hu, Y.; Kishi, Y. J. Am. Chem. Soc. 2012, 134, 6136-6139. doi:10.1021/ja302177z

30. Peng, J.; Kishi, Y. Org. Lett. 2012, 14, 86-89. doi:10.1021/ol202878v

31. Aicher, T. D.; Buszek, K. R.; Fang, F. G.; Forsyth, C. J.; Jung, S. H.; Kishi, Y.; Matelich, M. C.; Scola, P. M.; Spero, D. M.; Yoon, S. K. J. Am. Chem. Soc. 1992, 114, 3162-3164. doi:10.1021/ja00034a086

32. Namba, K.; Kishi, Y. J. Am. Chem. Soc. 2005, 127, 15382-15383. doi:10.1021/ja055966v

33. Kim, D.-S.; Dong, C.-G.; Kim, J. T.; Guo, H.; Huang, J.; Tiseni, P. S.; Kishi, Y. J. Am. Chem. Soc. 2009, 131, 15636-15641. doi:10.1021/ja9058475

34. Dong, C.-G.; Henderson, J. A.; Kaburagi, Y.; Sasaki, T.; Kim, D.-S.; Kim, J. T.; Urabe, D.; Guo, H.; Kishi, Y. J. Am. Chem. Soc. 2009, 131, 15642-15646. doi:10.1021/ja9058487

35. Liu, X.; Henderson, J. A.; Sasaki, T.; Kishi, Y. J. Am. Chem. Soc. 2009, 131, 16678-16680. doi:10.1021/ja9079308

36. Okude, Y.; Hirano, S.; Hiyama, T.; Nozaki, H. J. Am. Chem. Soc. 1977, 99, 3179-3181. doi:10.1021/ja00451a061

37. Chen, C.; Tagami, K.; Kishi, Y. J. Org. Chem. 1995, 60, 5386-5387. doi:10.1021/jo00122a011

38. Miller, J. J.; Sigman, M. S. J. Am. Chem. Soc. 2007, 129, 2752-2753. doi:10.1021/ja068915m

39. Miller, J. J.; Sigman, M. S. Angew. Chem., Int. Ed. 2008, 47, 771-774. doi:10.1002/anie.200704257

40. Harper, K. C.; Sigman, M. S. Science 2011, 333, 1875-1878. doi:10.1126/science.1206997

41. Harper, K. C.; Vilardi, S. C.; Sigman, M. S. J. Am. Chem. Soc. 2013, 135, 2482-2485. doi:10.1021/ja4001807

42. Huang, X.-R.; Chen, C.; Lee, G.-H.; Peng, S.-M. Adv. Synth. Catal. 2009, 351, 3089-3095. doi:10.1002/adsc.200900595

43. Chen, R.-Y.; Dhondge, A. P.; Lee, G.-H.; Chen, C. Adv. Synth. Catal. 2015, 357, 961-966. doi:10.1002/adsc.201400945

44. Chen, C. Synlett 1998, 1311-1312. doi:10.1055/s-1998-1984

45. Chopra, I.; Roberts, M. Microbiol. Mol. Biol. Rev. 2001, 65, 232-260. doi:10.1128/MMBR.65.2.232-260.2001

46. Xu, T.; Mu, X.; Peng, H.; Liu, G. Angew. Chem., Int. Ed. 2011, 50, 8176-8179. doi:10.1002/anie.201103225

47. Wang, F.; Qi, X.; Liang, Z.; Chen, P.; Liu, G. Angew. Chem., Int. Ed. 2014, 53, 1881-1886. doi:10.1002/anie.201309991

\section{License and Terms}

This is an Open Access article under the terms of the Creative Commons Attribution License

(http://creativecommons.org/licenses/by/4.0), which permits unrestricted use, distribution, and reproduction in any medium, provided the original work is properly cited.

The license is subject to the Beilstein Journal of Organic Chemistry terms and conditions:

(http://www.beilstein-journals.org/bjoc)

The definitive version of this article is the electronic one which can be found at:

doi:10.3762/bjoc. 12.275 